\title{
New Particle Simulation of the Magellanic Clouds
}

\author{
P.S. Li \\ University of Wyoming, P.O. Box 3905, University Station, Laramie, \\ $W Y$ 82 071 , USA
}

H.A. Thronson

Office of Space Science, NASA, Washington D.C. 20546, USA

\begin{abstract}
.
We use a modified TreeSPH code to simulate the MilkyWay-LMCSMC system using the latest observational results from Hipparcos on the proper motion of the Clouds. More than 120,000 particles are used for the simulation. Gravitational and hydrodynamic interactions among gaseous and stellar components in the system have been considered. The Magellanic Stream, some high velocity clouds, and the barred structure of LMC are reproduced. A leading gas arm from the SMC is predicted. From the simulation, the long term binary status of LMC-SMC is questioned as frequent encounters between the LMC and the SMC will have difficulty in explaining the survival of the SMC.
\end{abstract}

\section{Introduction}

The Magellanic Clouds are the two largest satellite galaxies of the Milky Way. The discovery of the Magellanic Stream provides direct evidence of interaction between the Clouds and the Milky Way. The previous simulations (e.g., Murai \& Fujimoto 1980; Gardiner \& Noguchi 1996) on this system did not include hydrodynamics, so cannot answer all the questions about the actual mechanism of forming the Magellanic Stream, the gas bridge between the LMC and the $\mathrm{SMC}$, and the origin of high velocity clouds. Also, gaseous exchange between the two Clouds is important to their structure and evolution.

We developed a parallelized TreeSPH code and used it to simulate the Milky Way-LMC-SMC system. Initial conditions of the 3 galaxies are obtained by backward integration using the latest observational results from Kroupa \& Bastian (1997) for the proper motion of the Clouds. A total of 116,736 particles (1/3 are gas particles) have been used. All three galaxies in the system are assumed to be disk galaxies. The mass ratio, including dark matter, of Milky Way:LMC:SMC = 1:0.02:0.003. In the model, the Milky Way has a very massive dark halo based on the latest estimation by Kulessa \& Lynden-Bell (1992). The simulation lasts an equivalent of 1.35 billion years. 


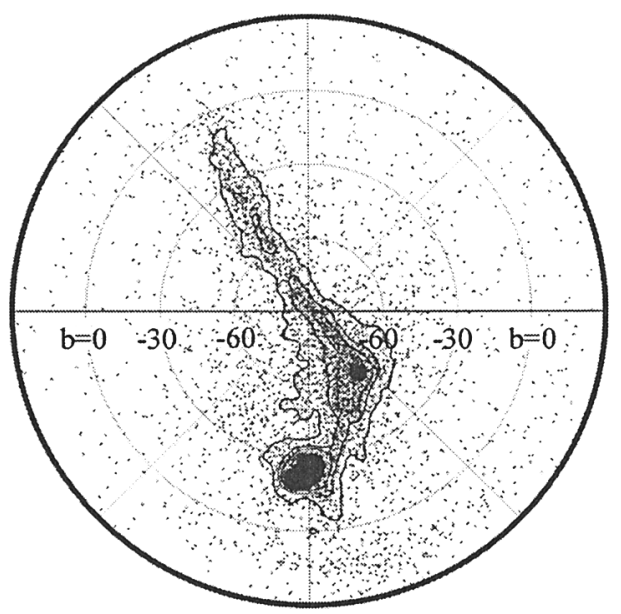

Figure 1. Column density of gas around the Magellanic Clouds and the Stream.

\section{Simulation Results and Conclusion}

We clearly rule out the Stream formation mechanism due to ram pressure from a halo of gas (Heller \& Rohlfs 1994) and reproduce most of the observed features, including the Magellanic Stream (see Fig. 1), high velocity clouds, barred structure of the LMC, and the leading gas arm from the SMC, which appears as the material bridge between the LMC and the SMC.

Some of the high velocity clouds are formed from the gas material of the lead gas arm and the SMC. These gas clouds are scattered and accelerated by the LMC during their encounter.

From the simulation, we find that the LMC and the SMC are not in a binary orbit at the beginning of the simulation. They came within $20 \mathrm{kpc}$ of each other about 550 million years ago and the encounter lasted for about 400 million years. During this encounter, more than half of the gas from the SMC is lost and the SMC is almost destroyed. The continued interaction between the LMC and the SMC will have difficulty in explaining the survival of the SMC.

Acknowledgments. This work is supported by the ISO program and the NCSA grant \#MCA94P001N, and is a part of the first author's Ph.D. Thesis.

\section{References}

Murai, T., \& Fujimoto, M. 1980, PASP, 32, 581

Gardiner, L.T., \& Noguchi, M. 1996, MNRAS, 278, 191

Heller, P., \& Rohlfs, K. 1994, A\&A, 291, 743

Kulessa, A.S., \& Lynden-Bell, D. 1992, MNRAS, 255, 105 


\section{Discussion}

Eric Maurice: What can you say about the thickness of the LMC disk after the encounter of the two Magellanic Clouds and of the Galaxy?

$L i$ : The thickness of the LMC disk is increased after the encounter with the SMC and the Milky Way. However, I haven't measured the actual value from the data yet.

Nolan Walborn: The LMC bar looks centered in the simulations. Can you comment on why the actual bar is eccentric?

$L i$ : Some of the participants have asked about this question in the last few days. Unfortunately, we haven't investigated this question in detail yet. It is hard to tell if the bar is centered with the stellar disk from the animation. We will look at this problem to find out if the Milky Way is the cause of the offset of the LMC bar as soon as possible.

William Kunkel: What is the bright blob flying off the gas disks?

$L i$ : Some of the gas from the SMC and the leading arm are scattered and accelerated by the LMC and fall back in the direction of the Milky Way. The masses of these gas blobs are in the range of $10^{6}$ to $10^{7} \mathrm{M}_{\odot}$. They could explain the origin of some of the high velocity clouds observed. 